\title{
Gradiente alveolo-arterial de oxígeno en los enfermos con EPOC
}

El aparato respiratorio, como tal, cumple como principal misión el intercambio de gases. Esto es posible merced a tres factores:

1. Factor pulmonar, representado por la movilidad respiratoria que permite la ventilación pulmonar y con ello la captación del oxígeno del medio externo y la expulsión del carbónico. Además hay que valorar la perfusión pulmonar y una especial referencia a la zona alveolo-capilar donde se desarrolla la transferencia gaseosa.

2. La circulación sanguínea impulsada por la bomba cardiaca que la moviliza desde cualquier órgano de la economía hasta los capilares pulmonares donde se llevara a cabo la hematosis.

3. Los hematíes, que con su contenido en hemoglobina, representan el principal vehículo para el transporte del oxígeno.

Un fallo en cualquiera de los tres sistemas, como puede deducirse, supondrá un trastorno mayor o menor en el equilibrio que debe existir para el normal movimiento de los gases. Considerando que la circulación y la sangre reúnen condiciones aceptables debemos prestar atención especial a la ventilación pulmonar y en este sentido dos datos sobre el normal fisiologismo respiratorio como recuerdo:

1. Durante la respiración, se establece el intercambio de gases, pasando el $\mathrm{O}_{2}$ al interior del capilar y eliminándose el $\mathrm{CO}_{2}$. Esta transferencia se realiza por difusión, merced a la tendencia de las moléculas a moverse desde una región de mayor concentración a otra con menor concentración de gas, situación regulada por la ecuación de Fick:

$$
\mathrm{V} \text { gas }=(\text { área } / \text { grosor }) .(\mathrm{P} 1-\mathrm{P} 2) . \mathrm{D} \text { : }
$$

La cantidad de gas que difunde (Vgas) es inversamente proporcional al grosor del área que ha de salvar y directamente proporcional a la superficie de intercambio y a la diferencia de presiones entre el alveolo (P1) y el capilar (P2) y a una constante de difusión (D) que a su vez es directamente proporcional a la solubilidad del gas e inversamente proporcional a la raíz cuadrada de su peso molecular. Esto implica que el $\mathrm{CO}_{2}$ difunda unas 20 veces mas rapido que el $\mathrm{O}_{2}$ ya que su solubilidad es mayor.
2. La sangre procedente de la periferia a través de la arteria pulmonar y sus ramas llega al capilar venoso con una $\mathrm{paO}_{2}$ de 40 y una $\mathrm{paCO}_{2}$ de 45. A nivel alveolar la $\mathrm{pAO}_{2}$ es aproximadeamte de 100 y la $\mathrm{PACO}_{2}$ de 40 , lo que facilita el normal intercambio. Entre el alveolo y el capilar existe una fina membrana de separación que apenas influye en el intercambio en condiciones de normalidad y asimismo es valorable comentar las diferencias fisiológicas que existen en el cociente V/Q a nivel de vértices y bases pulmonares al tener mayor ventilación los vértices y mayor vascularización las partes bajas de ambos pulmones por efecto de la gravedad, sin que este punto suponga tenga significación clínica a la hora de valorar el promedio en la transferencia de los gases.

Aunque tal como hemos comentado la difusión se realiza pasivamente equiparándose los niveles a uno y otro lado de la membrana, existe, en condiciones normales, una diferencia de presión entre el oxígeno alveolar y el del capilar pulmonar arterializado: es lo que se ha venido en llamar gradiente alveolo-arterial de oxígeno (A-a) $\mathrm{O}_{2}$.

En condiciones normales esta justificado por:

- La resistencia que ejerce la citada membrana alveolocapilar al paso del $\mathrm{O}_{2}$ que con la edad se incrementa a valores que debemos considerar.

-Zonas de pulmón en situación de reserva funcional con una relación V/Q inferior a la normal, lo que supone un pequeño efecto shunt.

-Mezcla de sangre venosa procedente de las venas bronquiales y del corazón (venas de Tebesio) que drenan en las venas pulmonares portadoras ya de sangre oxigenada.

El gradiente alveélo-arterial de oxígeno permite una evaluación más completa e integrada de la posible alteración del intercambio pulmonar de gases. Puede calcularse según las siguientes expresiones:

$\mathrm{A}-\mathrm{aO}_{2}=\mathrm{PAO}_{2}-\mathrm{PaO}_{2}$, siendo la $\mathrm{PAO}_{2}$ :

$\left.\mathrm{PAO}_{2}=\mathrm{PIO}_{2}-\mathrm{PaCO}_{2}\left(\mathrm{FiO}_{2}+\left[1-\mathrm{FiO}_{2}\right) / \mathrm{CR}\right]\right)$.

Donde $\mathrm{PAO}_{2}=$ Presión alveolar de $\mathrm{O}_{2}$

$\mathrm{PIO}_{2}=$ Presión parcial de $\mathrm{O}_{2}$ en el aire inspirado

$\mathrm{FiO}_{2}=$ Fración de $\mathrm{O}_{2}$ inspirada $(0,21)$

$\mathrm{PaCO}_{2}=$ Presión arterial de carbónico

$\mathrm{CR}=$ Cociente respiratorio $(0,8)$ 
o bien la ecuación simplificada:

$\mathrm{PAO}_{2}=\mathrm{PIO}_{2}-\mathrm{PaCO}_{2} / \mathrm{CR}$

El gradiente alveolo-arterial de $\mathrm{O}_{2}$ se describe como el medio generalmente aceptado para diferenciar las hipoxemias debidas a hipoventilación (por problemas de los centros respiratorios, musculares, del esquéleto torácico, etc.) de las causadas por enfermedades que afectan al parénquima pulmonar. La hipoventilación aumenta el $\mathrm{CO}_{2}$ a nivel alveolar y arterial mientras que el $\mathrm{O}_{2}$ está disminuido en ambos compartimientos, pero manteniendo la relación y por consiguiente el gradiente $\mathrm{A}-\mathrm{aO}_{2}$ no se modifica.

En la EPOC, las alteraciones de la distribución, además de la afectación de la membrana de separación alveolo-capilar justifican la dificultad para el intercambio de gases elevándose el gradiente. Es lo que habitualmente sucede. No obstante, en ocasiones, pueden presentarse excepciones a esta norma justificando un posible error:

-En pacientes que presentan marcada hipercarbia, efectivamente, el gradiente puede ser normal y esto porque el $\mathrm{A}-\mathrm{aO}_{2}$ y la $\mathrm{PaCO}_{2}$ se correlacionan de forma inversa, de tal forma que sujetos con intensa hipercapnia el $\mathrm{A}-\mathrm{aO}_{2}$ puede presentarse falsamente dentro de la normalidad; la existencia de un A$\mathrm{aPCO}_{2}$ elevado en pacientes con EPOC puede infraestimar el cálculo del $\mathrm{A}-\mathrm{aPO}_{2}$, al asumir que la presión alveolar del carbónico debe ser igual a la capilar.

\section{Bibliografía}

1. Fhari. A theorical analysis of the alveolar-arterial $\mathrm{O}_{2}$ difference with special reference to the distribution effect. J Appl Physiol 1955; 7:599-602.

2. Gray BA. Interpretationof the alveolar-arterial oxygen difference in patiens with hypercapnia. Am Rev Respir Dis 1991; 143: 4-8.

3. Mellemgaard K. The aalveolar-arterial oxigen difference: its size and components in normal man. Acta Physiol Scan 1966; 67:10-20.

4. Shapiro BA. Manejo clínico de los gases sanguíneos, $4^{\mathrm{a}}$ edicion. Buenos Aires: Panamericana 1996; 157.
-Para eliminar en lo posible el error en la interpretación del gradiente de $\mathrm{O}_{2}$ es importante hacer el cálculo con la fórmula completa antes citada. Eliminamos así, con este proceder, aunque sea más engorroso, gran número de casos de EPOC que podrían estar mal diagnosticados al aplicar la fórmula simple.

-El cociente respiratorio es otra variable a considerar, si bien habitualmente no se calcula por no hacer demasiado complicada la fórmula y porque tampoco implica mayor relieve en la interpretación de los resultados.

-En la EPOC, pues, la alteración del gradiente alveolocapilar de $\mathrm{O}_{2}$ es la norma, tal como sucede en el resto de enfermedades con afectación del parénquima pulmonar. En algunos casos con carbias muy elevadas puede ser normal, máxime si aplicamos la fórmula simplificada para hallar la presión alveolar de $\mathrm{O}_{2}$, pero no por ello debemos desestimar este dato como un dato más en el diagnóstico de esta enfermedad.

\section{S. A. SANTAMARÍA HERRERO}

Servicio Neumología.

Hospital Universitario Gregorio Marañón. Madrid

5. Skorodin MS. Respiratory diseases and A-a gradient measurement JAMA 1984; 252:1344.

6. De Vega A. Test de función pulmonar en las alteraciones del intercambio de gases. Rev PAR 1990; 95: 45-54.

7. Conroe JH. Fisiología de la respiración. Ed. Interamericana.

8. Carrión Valero F, Martínez Francés M, Perpiñá Tordera M, Pascual Izuel J.M. ${ }^{a}$, Marín Pardo J. Gradiente alveolo arterial de $\mathrm{O}_{2}$ en la EPOC con hipercapnia. An Med Interna (Madrid) 2001; 18 117-120. 